\title{
An Overall Perspective of Machine Translation with its Shortcomings
}

\author{
Alireza Akbari \\ Foreign Languages Faculty, University of Isfahan \\ PO box 81746-73441, Isfahan, Iran \\ E-mail: Dictogloss@gmail.com
}

Received: 05-10- 2013

doi:10.7575/aiac.ijels.v.2n.1p.1

Accepted: 15-11-2013

Published: 31-01-2014

URL: http://dx.doi.org/10.7575/aiac.ijels.v.2n.1p.1

\begin{abstract}
The petition for language translation has strikingly augmented recently due to cross-cultural communication and exchange of information. In order to communicate well, text should be translated correctly and completely in each field such as legal documents, technical texts, scientific texts, publicity leaflets, and instructional materials. In this connection, Machine translation is of great importance in translation. The term "Machine Translation" was first proposed by George Artsrouni and Smirnov Troyanski (1933) to design a storage design on paper tape. This paper sought to investigate an overall perspective of Machine Translation models and its metrics in detail. Finally, it scrutinized the ins and outs shortcomings of Machine Translation.
\end{abstract}

Keywords: language translation, Machine translation, MT models, MT metrics, MT shortcomings

\section{Introduction}

\subsection{History of Machine Translation (MT)}

In seventeenth century, translation of languages would be considered a dream. But in twentieth century, this dream came true. The process of translation which regarded as translation product was carried out by computer programs. Machine translation is not only did relate to the abstract intellectual exploration but also the application of computer to the amelioration of practical needs. The history of Machine Translation traces back to the 1950s and 1960s. In the mid1960s, the influence of ALPAC report would be observed, later the regeneration in the 1970s, the advent of operational and practical system in the 1980s, and new trends in research in the 1990s are the most brilliant periods in Machine Translation.

\subsection{Some Rudimental Traits}

Basically, Machine Translation alludes to computerized system of translation responsible for translation product with or without the help of human being which was later labeled as "Medium Restricted Theories ${ }^{1}$ (Munday, 2008, p. 11)". One of the important notes is the exclusion of computer-based translation devices and remote terminology databanks from Machine Translation. It should be noted that the limitation between machine aided human translation and human aided machine translation are in state of influx. Totally, it is better to say Machine Translation covers the translation process called "translating".

In practice, the ultimate aim of Machine Translation may be to produce high-quality translation; but the intended translation needs to be revised or post edited at the end of the process in order to saturate any tastes of various audiences. The important note in the previous sentence will be the word "post editing". It is better to say that Machine Translation cannot be able to exclude human assistance at all for which the process of post editing will be done by human in this connection. However, the numbers and the sorts of errors done by the MT are to a great extent different from that of human translation. Therefore, post editing stage will be of great importance in general. Considering the term "decoding ability" (Eugene Nida, 1964, p. 156-71) in Machine Translation is important to scrutinize. Whenever the main audience of the text is of various people, post editing stage will be of great importance. But assume that the primary audience is the specialist in a special field of study. In certain conditions, the output of Machine Translation may be unrevised if it is considered for specialists.

The translation quality of Machine Translation will be ameliorated by utilizing more translation methods and stipulating some more limitations on input. The first Machine Translation was concocted for special aims. For example, METEO was regularly translating around 45000 words of weather bulletins every day, from English into French for transmission to press, radio, and television. In this essence, all of the intended words were related to the source language which restricts the range of vocabulary, hyponymy, monosymy, polysemy, and complex grammatical structures in the target language. Somehow, it is better labeled as direct translation or generally word for word rending. Machine Translations are designed for bilingual systems and polygonal languages. For example, SYSTRAN's software renders a source language text into 52 languages for individuals in order to saturate any taste of comprehension. In bilingual system of Machine Translation, natural equivalence or one to one correspondence will be of great importance (i.e. $\mathrm{A} \rightarrow \mathrm{B}$, and 
$\mathrm{B} \rightarrow \mathrm{A}$ ). But for the latter case, the translation will be done in polygonal languages unidirectionally (i.e. $\mathrm{A} \rightarrow \mathrm{A}^{\prime}, \mathrm{A}^{\prime \prime}$, $\left.\mathrm{A}^{\prime \prime \prime}\right)$. In this case, the equivalence will be directional one and Machine Translation cannot exoticize word for word rending.

Interlingua approach is the other strategy which is completely utilizable in this field. In Interlingua approach, source language text converts into representations to more than one language such as SYSTRAN. In this connection, Machine Translation plays the mediation ${ }^{3}$ role (Hatim and Mason, 1997) in which an SL text transmits to Interlingua approach and then converts into the TL text. One of the differences among direct translation and Interlingua approach will be of economy of language in a multilingual conditions. For direct translation, translation requires $\boldsymbol{n}(\boldsymbol{n}-\mathbf{1})$ bilingual system, whereas in Interlingua approach will be $\boldsymbol{2} \boldsymbol{n}$ interlingual programs. Economy of language alludes to utilizing more than three languages in general. In some occasions, Interlingua approach is based on manipulative and auxiliary language such as Esperanto.

Interlingua approach of Machine Translation system consists of three stages: (1) analysis and representation of source text syntactic structure; (2) transfer into target structure; and (3) synthesis of output from that structure (Somers, 1998, p. 145). In transfer approach, Machine Translation systems scrutinize the discrepancies and ambiguities in the language inherently. In the scope of analysis and syntactic structure, different levels of linguistic descriptions will be used such as syntax, semantics, and morphology. Syntactically speaking, Machine Translation utilizes syntactic analysis such as subordinations, conjunctions, phrase structures, and dependency rules. In semantic view, MT analyzes semantic analysis such as functional, structural, and lexical ambiguities. In scope of morphology, Machine Translation peruses morphological analysis such as compound words, complex words, and word endings. The last stage of Machine Translation systems deals with output or translation product of intended text carried out in first and second stages of Interlingua approach.

To make the long story short, Machine Translation systems scrutinize into two generations. The first generations of Machine Translation deals with "direct" translation systems since they are fundamentally word based, each ST word will be look up and replace by corresponding TL term. The reputation of MT first generation fell down in 1960s. Instead the concentration of Machine Translation systems inclines to language processing known as computational linguistics. The second generation of Machine Translation systems deals with "indirect" translation which adds an intermediate stage between ST and TT phase. The indirect translation includes two stages: (1) transfer approach and (2) Interlingua approach. In these two stages, the ST meaning is represented in an abstract form before being reconstructed in the TT. These three stages of second generation of Machine Translation can be summarized in "Bernard Vauquois' Pyramid":

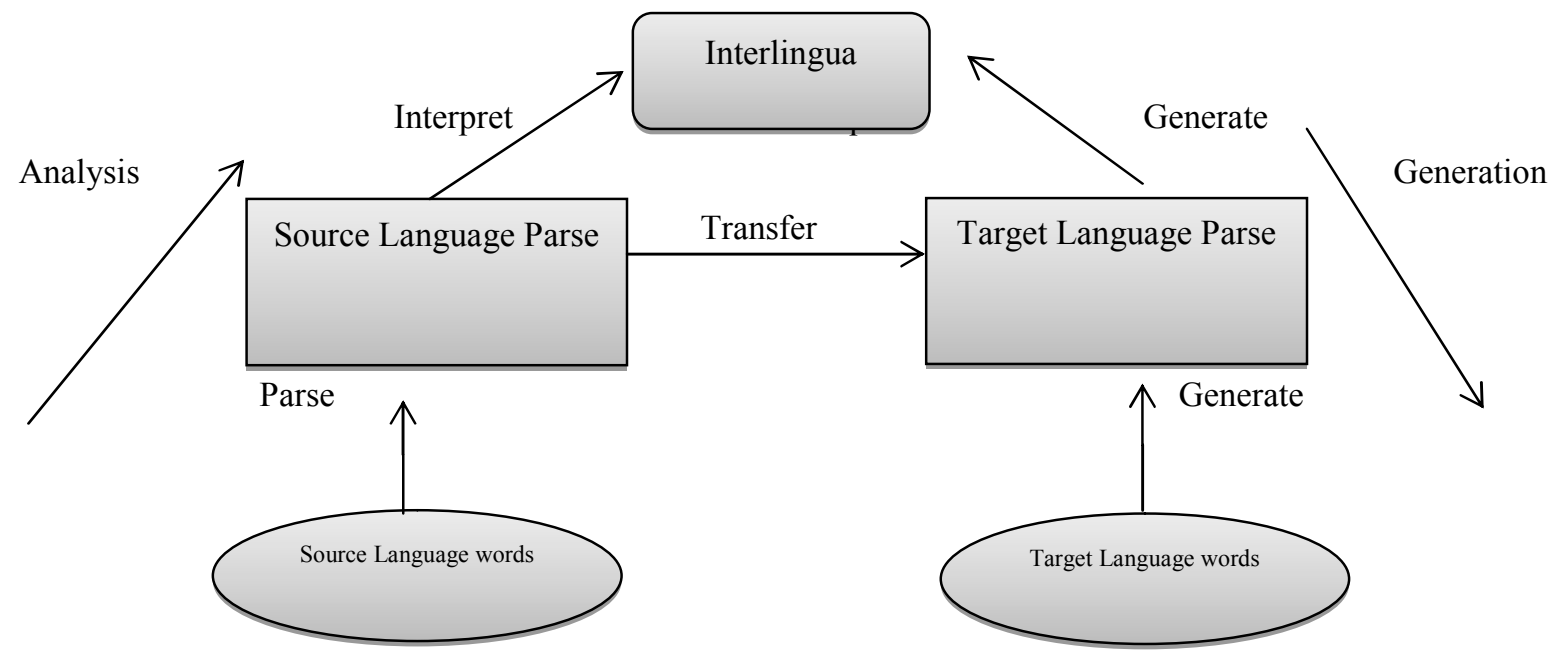

Figure1. Bernard Vauquois' Pyramid

\subsection{Pioneers of Machine Translation (MT)}

The first concrete proposals of Machine Translation programs were presented by George Artsrouni and Peter Smirnov Troyanski in this connection. Artsrouni manufactured a storage device on paper tape which could be utilized for searching any equivalents in other languages. The prefabricated device of Artsrouni's was demonstrated in 1937. He could envisage Machine Translation in three stages: (1) an editor of the source language securitizes each word logically into its base form and syntactic function; (2) the second stage was the transferring the input of the source language to the target one; and (3) another editor in target language inspects and converts the intended translated text in accordance with the situation and environment of the target language. It should be noted that the first Machine Translation system considered both bilingual and multilingual environment of translation. In this direction, he believed in mechanization of logical analysis.

A few years later, a revolution in American universities such as University of California, Los Angeles University, and Massachusetts Institute of Technology took place. In 1951, MIT appointed some full time researchers in analyzing 
Machine Translation in details. Amongst these researchers, Yehoshua Bar-Hillel criticized Machine Translation in his Report on the State of Machine Translation in the United States and Great Britain on 1959. He then argued that the text should be written in MT-oriented and construction of sub-language systems. But in this direction, human assistance would be of great importance to produce the source language text and then revise it in the target language.

In the 1950s and 1960s, the focus of research in Machine Translation was on trial and error approaches which applied statistical methods and theoretical approaches to inspect linguistic research. But these approaches were completely time-consuming and it had to be concocted some advanced hardware to process every stage fast with less errors in general. Therefore, some of the researchers concentrated on trial and error approach and some on theoretical aspects on Machine Translation systems. Erwin Reifler at the University of Seattle condensed the direct translation or word for word approaches which included the construction of large bilingual lexicons at which lexicographic information was used both for choosing near equivalents and figuring out grammatical problems. In 1958, Gilbert King at IBM Corporation installed a system for US Air Force which generated and utilized translations for many years. But in this direction, his system had some deficiencies such as crude and unintelligible output. Therefore, Michael Zarechnak at Georgetown University presented a more eclectic approach which scrutinized empirical analysis of the text which later called Georgetown Automatic Translation (GAT). This approach covered three aspects of Machine Translation: (1) morphological which dealt with rendering expressions and idioms; (2) syntagmatic which dealt which adjective modifications and agreement of nouns and adjectives; and (3) syntactic which inspected subject-verb agreement, sentence analysis, and relationships between clauses.

In 1958, The Linguistic Research Center (LRC) was established by Winfried Lehmann. LRC focused on syntactic research between English and German languages in general. Lehmann took an attempt to devise a Machine Translation for reversible grammars to achieve bidirectional renderings.

Last but not least, Machine Translation studies had been established in most of the European countries such as Belgium, Germany, France, and Hungary. Some of these Machine Translation methods were short-lived and could not deal with near equivalents in different translational situations and some had long-lived periods to translate the main essence of the text in general. But the most important factor is that all these Machine Translation methods, either old or new, suffers from some sorts of shortcomings which later this paper expounds them ins and outs.

\section{Review of Literature}

\subsection{Types of Machine Translation (MT)}

In a paper titled "Theoretical Overview of Machine Translation", Cheragui (2012) expressed Machine Translation into four categories:

\subsubsection{Machine Translation for Watcher (MT-W)}

This is a kind of Machine Translation which makes it possible for the readers who wanted to gain access to some information written in the target language. The intended Machine Translation was utilized to render military technological deeds. This Machine Translation was completely based on dictionary or word-based translation and was definitely different from linguistics-based Machine Translation in this connection.

\subsubsection{Machine Translation for Revisers (MT-R)}

This sort of Machine Translation aims at producing rendering with high quality of comparison to that of the first draft produced by human translation. The kind of rendering carried out in this Machine Translation can be labeled as "brush up" rendering which is completely applicable just for revisers. Here "reviser" means translator of the target language. The translator acts the role play both as renderer and reviser to make the intended text completely agreeable to the norms and disciplines of the target text.

\subsubsection{Machine Translation for Translators (MT-T)}

This kind of Machine Translation helps the translator to find online dictionaries and translation memory tools. But the most important factor in "Machine Translation for Translator" is PC-based translation tools. Homedian (1998) expressed that "tools for individual translators have been available since the beginning of office automation".

\subsubsection{Machine Translation for Authors (MT-A)}

This type of Machine Translation causes authors to have their texts translated into one or several languages. Therefore, it can be labeled as "Interactive MT" which considers the interaction among analysis and transfer of the text and not toward the author of the text. To put it in a nutshell, Homedian (1998) expressed that there have been no operational successes of MT-A; nevertheless, the designs of this Machine Translation more inclines to user-oriented.

\subsection{Some Metrics of Machine Translation}

\subsubsection{Bilingual Evaluation Understudy (BLEU)}

Papineni (2002) proposed one of the first automatic measurements as a reference for translation evaluation in accordance with some rules and disciplines. The fundamental aspect of BLEU Machine Translation was to juxtapose the degree of similarity amongst Machine Translation and two or more renderings completed by reference translation based on $\mathrm{n}$-gram ${ }^{4}$ precision. The formula of BLEU Machine Translation is as follows:

$$
B L E U=B P x_{e}\left(\sum_{n=1}^{\mathrm{n}} w_{i} \log p n\right)
$$

It should be noted that the ranking behavior is more apparent in the Log domain, therefore; 


$$
\log B L E U=\min \left(1-\frac{r}{e}, 0\right)+\sum_{\mathrm{n}=1}^{W} w_{n} \log p_{n}
$$

In this connection, $\mathrm{N}=4$ as the baseline, and the uniform weights $\mathrm{w}_{\mathrm{n}}=1 / \mathrm{N}$

Pn: refers to he number of n-gram which is present in one or more reference translation, and then divided by the total of n-gram of MT

$$
\begin{aligned}
& p_{n}=
\end{aligned}
$$

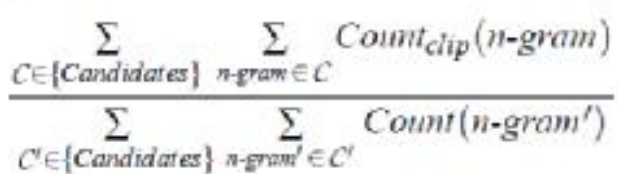

$\mathrm{w}_{\mathrm{i}}$ : refers to positive weights (in some conaltion, tne intenaea term callea $\mathrm{w}_{\mathrm{n}}$ )

BP: alludes to brevity penalty which penalize renderings as short translation. The formula for calculating "brevity penalty" will be as follows:

-c: refers to the length of Candidate Transl

$$
13 \mathrm{p}\left\{\begin{array}{lll}
1 & \text { Si } & \mathrm{c}>\mathrm{r} \\
e^{1-\frac{r}{r}} & \text { Si } & \mathrm{c} \leq \mathrm{r}
\end{array}\right.
$$

-r: alludes to Effective length of reference translation

In BLEU Machine Translation, there exists two metrics: (1) the reliance on higher n-grams, and (2) brevity penalty.

\subsubsection{Word Error Rate (WER)}

This metrics proposed by Popovic and Ney in 2007. This one rudimentally utilized for Automatic Speech Recognition and then juxtaposes the Sentence Hypothesis to a Sentence-based on the Levestein distance. In this connection, the above-mentioned metrics was used to evaluate the quality of translation hypothesis in a relation to reference translation. In this track, this metric calculates the minimum number of edits such as deletion, substitution, elision, and insertion of the word into the sentence. These edits are used to procure the good translation and be identical to that the reference translation. The formula of this metrics is as follows:

$$
W E R=\frac{1}{\text { Wref }} x d_{L}(\text { ref, hyp })
$$

$-d_{L}$ (ref,hyp): refers to the Levenstein distance amongst the reference translation "ref" and the hypothesis translation "hyp".

$-\mathrm{N}_{\text {ref: }}$ alludes to the size of the reference translation

In this track, if one researcher wants to calculate WER decomposition over $\operatorname{POS}^{5}$ class, he or she should obey the next metrics which is:

$$
\operatorname{WER}(p)=\frac{1}{\operatorname{Nref}} \sum_{k=1}^{k} n\left(p, \operatorname{err}_{k}\right)
$$

$-\mathrm{n}\left(\mathrm{p}, \mathrm{err}_{\mathrm{k}}\right)$ : refers to the number of errors in $\operatorname{err}_{\mathrm{k}}$ procured by words in POS class.

As an example in "Word Error Rate: "Decomposition over POS Classes and Applications for Error Analysis" Popovic and Ney (2007, p.49-51) reveal that the standard WER of the whole sentence will be $4 / 12=33.33 \%$. The contribution of noun is:

Table 1. Example for illustration of Actual Error

\begin{tabular}{|l|}
\hline Reference \\
\hline Mister\#N Commissioner\#N , \#PUN \\
\hline Twenty-four\#Num hours\#N \\
\hline sometimes\#ADV can\#V be\#V too\#ADV \\
\hline much\#Pron time\#N . \#PUN \\
\hline Hypothesis \\
\hline Mrs\#N Commissioner\#N , \#PUN \\
\hline Twenty-four\#Num hours\#N is\#V \\
\hline Sometimes\#ADV too\#ADV \\
\hline much\#Pron time\#N . \#PUN \\
\hline
\end{tabular}


Table 2. WER Error

\begin{tabular}{|l|l|l|}
\hline Reference & $\begin{array}{l}\text { Hypothesis } \\
\text { Error }\end{array}$ & $\begin{array}{l}\text { Error } \\
\text { Type }\end{array}$ \\
\hline Mister\#N & Mrs\#N & Substitution \\
\hline sometimes\#ADV & is\#V & Substitution \\
\hline can\#V & & Deletion \\
\hline be\#V & sometimes\#ADV & Substitution \\
\hline
\end{tabular}

Then:

WER $(\mathrm{N})=1 / 12=8.3 \%$, of verbs WER $(\mathrm{V})=2 / 12=16.7 \%$ and of adverbs WER $(\mathrm{ADV})=1 / 12=8.3 \%$

\subsubsection{Position Independent Word Error Rate (PER)}

In 1997, Tillman proposed the metrics of Per. In this connection, PER juxtapose the number of words of MT to those of the reference translation. The important consideration in this metrics will be the unimportance of the word sequence in the sentence. The formula of the indented metrics is as follow:

$$
P E R=\frac{1}{\text { Wref }} x d_{\text {per }}(\text { ref, hyp })
$$

$-\mathrm{d}_{\mathrm{per}}$ : refers to the difference amongst the occurrence of words in MT and the reference translation.

In this regard, if one researcher wants to calculate PER decomposition over POS class, he or she should obey the next metrics which is:

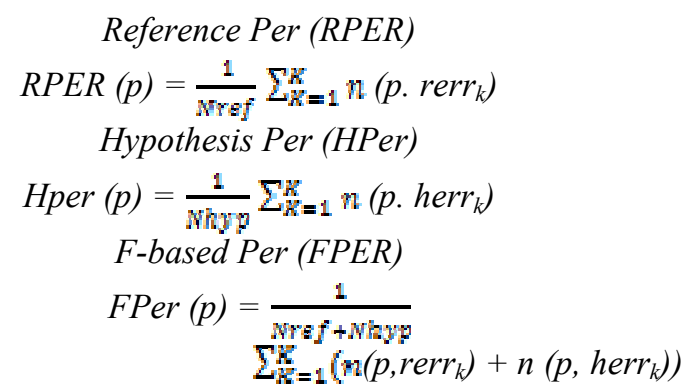

\subsubsection{Translation Error Rate (TER)}

In 2006, Snover proposed the TER metrics. He defined TER as the minimum number of edits needed to change a hypothesis so that it exactly matches one of the references. Translation Rate Error involves insertion, deletion, substitution of the words, and sequence of contiguous words. In this metric, the researcher just measures the number of edits to the nearest reference. The formula of the intended metric is as follow:

$-\mathrm{Nb}$ (op): alludes to minimum number of edits

$$
T E R=\frac{\text { Ma }(\text { ogs })}{\text { Arreg } \text { Wref }}
$$

-Avreg $\mathrm{N}_{\text {Ref: }}$ refers to the average size in words references

\section{Methodology}

\subsection{Shortcomings of Machine Translation}

This section deals with the shortcomings of Machine Translation (MT) in details. It is completely natural to express that in a translation program, few phases have to be taken relied on the perception of Machine Translation either directly or indirectly, but unfortunately MT will not be able to operate well and the whole program will come to a deadlock. Therefore, it is better to expatiate the shortcomings of Machine Translation in detail.

\subsubsection{Syntactic Problem}

One of the most important aspects of Machine Translation will be operational syntax. In syntactic structure, Machine Translation should be paid attention to both source language and target language. But, not every structure of language obeys the same method of forming clauses, phrases, and sentences. For example, English has the system of SVO in forming sentences in general, but languages such as Persian and Japanese have the system of SOV in forming the sentences. It is thoroughly an arduous task to match the two different structures of languages to make an understandable rendering to saturate every taste of audience. "Meaning of pronoun" in different situation is the other Achilles Hills of Machine Translation which cannot be able to distinguish the right pronoun. For example, the pronoun "you" in English is used for both singular and plural. But, the other languages such as the German and Italian languages have their own system of pronoun and express the second singular pronoun as "du and tu" respectively. How will it be possible for Machine Translation to ascertain the right gist of translator? According to Yehoshua Bar-Hillel (1953, p. 218), "a student of chemistry who has, either in his mind or on his shelves, a complete description of all known methods of synthesizing or otherwise producing them, would be at the complete loss when presented with the task of determining the composition of an unknown mixture of substance." To cut the story short, lots of new vocabularies and structures will add to the every language nowadays. What is happened if the intended Machine Translation will not be able to be upgraded? It can be stated that, Machine Translation cannot render any technological vocabularies unless it is upgraded. 
The other problem to mention will be "structural ambiguity" in Machine Translation. In some situations, one sentence can be inferred in more than one way and it makes translation hard to grasp. For example, "John mentioned the notebook I dispatched to Jessica". This sentence can be rendered in two ways: (1) John mentioned the notebook which I dispatched to Jessica, and (2) John mentioned to Jessica the notebook which I dispatched. It should be stated that Machine Translation will not be dealt with these structural problems and it cannot saturate the taste of audience completely. As another example, "pregnant women and children" is translated by Machine Translation as "le donne incinte et le bambine incinte" which is completely wrong and it is actually hard to perceive. This sentence expresses that only women are pregnant. It should be noted that Machine Translate cannot be able to distinguish the context of the sentences. Therefore, MT matches the entire items in a sentence. The term "Machine Translation" can be rendered as a rickety bridge in translation and it is used just for comprehending the gist of the target language text in general. Most of the problems arise when the Machine Translation wants to render the phrase of "es gibt" in the German Language. The German word "es" has its English equivalents as "it", "he", "she", and the German ward "gibt" has the equivalent of "gives". This case is called "over-differentiation" in translation where the source language word has three or four equivalents in the target language text. How can Machine Translation deal with this occasion in general? Or vice versa, how can Machine Translation render "under-differentiation" in the target language? In most of the conditions, Machine Translation will deal with "one to one correspondence" or natural equivalents called literal translation of the words. To make a long story short, Machine Translation cannot perform well in these fields: (1) straightforwardness of translation, (2) the orders of the structure in source and target language, (3) paraphrasing of the text into intended languages, (4), and opting for the right structure in the target language text such as imperative mood in English infinitive in French (Antii IImo, 2003, p.15).

\subsubsection{Natural Language Intertranslatibility}

Natural language according to Webopedia is defined as "A human language. For example, English, French, and Chinese are natural languages. Computer languages, such as FORTRAN and $\underline{\mathrm{C}}$, are not. Probably the single most challenging problem in computer science is to develop computers that can understand natural languages. So far, the complete solution to this problem has proved elusive, although a great deal of progress has been made. Fourth-generation languages are the programming languages closest to natural languages." According to this definition, all natural language are universal. Natural language includes two senses: (1) closed language is a subset of natural language whose rules and structures both syntactically and semantically emanate from the behavior of the users at the specific time. (2) Open language is the formal language of the author in general. This sort of language amalgamates the grammar of an object-centered programing language with the natural language universality (Yehoshua Bar-Hillel 1953, p. 219). How is natural language Intertranslatibility related to shortcoming of Machine Translation? As it was states earlier in this section, natural language dealt with senses of the source and target language. It should be noted that Machine Translation cannot render senses of the text in different situation. Machine Translation will not be able to contain indexical expression. Indexical expressions are expressions whose forms have characteristics which are only associated in nature with its meaning. For example, if the source language text aims to convey the exact sense of the sentence "I am thirsty" in target language, how can Machine Translation succeed to transfer the sense of the sentence completely? To what extent is the person thirsty? Or how can it be possible to render the poem via Machine Translation? How can it be possible to keep just one signifier and one signified in Machine Translation? How will it be possible to convey the exact sense of creative composition in poem rendering? And how is it possible to keep translation of the source and target language in "Gray zone" in order to saturate any taste of audience? Last but not least, to answer these questions, the translator should be aware of the source language context of the word sense in general in order to reduce the effect of the target language words for the target readers. He or she has to walk on the area between the two poles of source and target translation. To make the long story short, it can be stated that "Es ist die Zeit, um die Parallelen Straßen der Übersetzung zu verschmelzen und gelangen an die Tür der Unendlichkeit (Akbari, 2011)." (It's the time to amalgamate the parallel roads of translation and reach to the door of infinity).

\subsubsection{Idioms, Slangs, and Expressions}

In the field of linguistics, there are two kinds of meanings: (1) Primary meaning which is related to the first meaning of the word comes to mind of the reader. Machine Translation can be able to get along with kind of meaning because the rudimental task of Machine Translation is to render one to one correspondence in general. According to Yule (2006, p. 100) "conceptual (primary) meaning covers those basic essential components of meaning that are conveyed by the literal use of the word." It can be stated that in translating such words, Machine Translation will not encounter any problem transferring the main essence of the text. (2) Associative meaning which is pertained to the stylistic or hidden meaning of the word. In this direction, different people might have different points of view of the intended word. Generally speaking, associative meaning is pertained to idioms and expression expressed in diverse conditions by the people. Idiom is defined by Merriam Webster (2013) "the language peculiar to a people or a district, community, or a class." Some of idioms are extremely pertained to the local color of source language and will not be found in the target language. And some of them are related to the target language only. In these conditions, Machine Translation cannot make a fix decision for translating such idioms and expression. How can MT transfer the local color of source language to the target one? How can Machine Translation convey the tone of voice of the idioms and expressions in different occasions? For example, in the French language, there is one expression as "C'est quoi, ce Bronx" which literally means "what is this, the Bronx?" In this regard, the target reader cannot perceive the associative meaning of the intended idiom. The figurative or associative meaning of the intended expression will be "what's a dump" which is completely obvious for the target reader. Some critics express that the only way of rendering idioms in Machine Translation is not 
to have idioms at all. It means that the source language should utilize the direct language in Machine Translation in order to see any problem for the target reader. All these cases are true of "Slang". Slangs are any kinds of expression which are spoken by laymen and less-educated people in general. The power of Slang in transferring the main essence of the text is higher than that of idiom. For example, "story vomit" cannot be rendered directly in Machine Translation because it causes the creation of an artificial meaning of this phrase. In an article titled as "Exploring into the New Model Procedure in Translation: Wafting as a case in Point" (Akbari, 2013, p. 19-20) he expressed that some words such as "Band", "Ticket", and "Girls" defined literally in the German Language and associatively rendered in the English Language. It means that, in most of the cases, the primary meaning is of great importance and the figurative meanings are less important in the German language. How can Machine Translation transfer this kind of situation in the English target language?

In solving this problem, there are two ways: (1) post editing which is done by the target translator being an expert in the source language. This work should be done cautiously because it causes the creation of multitudes of meanings and it makes translation ambiguous. (2) According to Yehoshua Bar-Hillel (1953, p. 222), changing the old forms or rules of the language and then adding some more new rules to the intended rule are good solutions in order to render a translated text via the new set defined in the old one. For the ease of the reader, the translator should define everything literally in accordance with natural equivalence or one to one correspondence.

\subsubsection{Lexical Ambiguity}

Lexical ambiguity is defined as source language renders as more than one equivalent in the target language. It should be noted that Machine Translation cannot distinguish the multiple meanings of the source language words in the target language. For instance, "river" can be rendered as "fleuve" and "rivere" in the target language. The multiplicity of meanings makes translation hard to perceive for Machine Translation. As another example, the word "light" can be translated as "clair", "facile", "lumière", and "feu" in target language. How can Machine Translation deal with this situation and how is it possible to saturate the needs of the reader in this connection? To solve such problems, contextual rules should be fed into Machine Translation in order not to encounter such issues. Machine Translation should set some pragmatic rules in different conditions in order to translate near to the source language text. It can be stated that "Rule-based Machine Translation", "Example-based Machine Translation", and "Statistical Machine Translation" solved these problems to some extent. Rule-based machine Translation (RBMT) is pertained to diverse kinds of linguistic rules. In the development of RBMT, two major paths are considered: (1) the direct approach which works amongst pairs of languages on the basis of bilingual dictionary. This approach renders words one to one correspondence without much detailed analysis of the syntactic and semantic structures of the target language. (2) The indirect approach which is more intricate in analyzing the syntactic and semantic structure of the target language and it is called the second generation of Machine Translation. Example-based Machine Translation (EBMT) is essentially based on the source language storage and alignment and this system also utilized the bilingual parallel corpora (Sergi Nirenburg, 1992, p48). In EBMT, translation is completed into three phase: (1) "Matching" which includes matches for the source language in the parallel corpora. (2) "Alignment" which includes distinguishing which parts of the translation is supposed to be reutilized. And (3) "Recombination" deals with amalgamation of those parts of the examples grammatically. And the last one is statistical Machine Translation (SMT) which is based on words and phrases or word sequences in a bilingual parallel corpus. These word sequences are pertained to word frequencies in this case. Whenever one word is on high level of sequence of utilizing by the target reader, it is in high level frequencies in translation. Therefore, SMT should scrutinize the frequencies and sequences of the words before the act of translation. But it should be noted that RBMT and EBMT are context bound and every step of translation should be pre-determined for these systems.

\subsubsection{Paralinguistic Problems}

When we communicate, we sometimes face problems in speaking and transferring the exact sense of our speech. For instance, we cannot recall the intended message or we need more time to design a new and intricate utterance. In these cases, these issues manifest paralinguistically. Paralinguistic features are the subpart of communication and do not include words. Facial expression, gestures, postures, and tone of voice are the examples of paralinguistic aspect of linguistics. It should be noted that paralinguistic features of the language are vehemently pertained to degree of frequency and intensification of the intended words in the target language. The best examples to consider will be "interjections". For instance, "Mmmm", "uh", "ouch", and "ooops" are the examples of interjection in linguistics. How can Machine Translation show the degree of intensification of the above-mentioned words correctly and rightly transfer the exact sense to the target language? How can Machine Translation show the uncertainty of the author of the text? How can Machine Translation be able to interpret the human behavior in diverse occasions? Interpreting human behavior is an arduous task in translation field. For instance, translating a poem of Shakespeare and then conveying the sense of that poem is beyond the ability of the normal renderer. How can the audience expect from Machine Translation to render correctly? In order to solve this problem, translators have proposed paralinguistic translation method.

According to Takatomo Kano (2012) Paralinguistic Translation Metric converts the source-side duration and mean power vector $\mathrm{X}$ into the target-side duration and mean power vector $\mathrm{Y}$ in accordance the following equation:

$$
\hat{Y}=\arg _{y} \max P(Y \mid X)
$$


In this equation, duration and power of each word using the source sided and power super is vector $\mathrm{x}_{\mathrm{i}}=\left[\mathrm{x}_{1}, \ldots \ldots \ldots, \mathrm{x}_{\mathrm{n}}\right]^{\mathrm{T}}$ and the target-side duration and power super is vector $\mathrm{y}_{\mathrm{i}}=\left[\mathrm{y}_{1}, \ldots \ldots \ldots, \mathrm{y}_{\mathrm{n}}\right]^{\mathrm{T}}$. If we conjecture that the duration and translation power of each word is self-reliant from that of the other word, the next equation will be as follows:

$$
\hat{Y}=\arg _{y} \max \prod_{i} P\left(y_{i} \mid x_{i}\right)
$$

$\mathrm{P}\left(\mathrm{y}_{\mathrm{i}} \mid \mathrm{x}_{\mathrm{i}}\right)$ is called acoustic exoticizing (word for word) translation and is defined in accordance with linear regression matrix which calculates as the following equation:

$$
\mathrm{P}\left(\mathrm{y}_{\mathrm{i}} \mid \mathrm{x}_{\mathrm{i}}\right)=\mathrm{N}\left(\mathrm{y}_{\mathrm{i}} ; \mathrm{W}_{\mathrm{ei}, \mathrm{ji}} \mathrm{x}_{\mathrm{i}}, \mathrm{S}\right)
$$

In this equation, $\mathrm{x}^{\prime}$ alludes to $\left[1 \mathrm{x}^{\mathrm{T}}\right]^{\mathrm{T}}$ and $\mathrm{W}_{\text {ei, ji }}$ refers to regression matrix. In this equitation, it should be considered the way of constructing the pair of the words. In order to do so, the equation of word pairs is as follows:

$$
\hat{\mathrm{W}}_{\mathrm{e}, \mathrm{j}}=\arg _{\mathrm{wi}, \mathrm{j}} \min \Sigma_{\mathrm{m}=1}^{N}\left\|\mathrm{y}^{*}{ }_{\mathrm{n}}-\mathrm{y}_{\mathrm{n}}\right\|^{2}+\alpha\left\|\mathrm{W}_{\mathrm{e}, \mathrm{j}}\right\|^{2}
$$

In this equation, $\mathrm{N}$ refers to the number of training samples, $\mathrm{n}$ alludes to identification of each training samples, $\mathrm{y}^{*}$ refers to the target language, and $\alpha$ alludes to a hyper-parameter to prevent over-fitting. It is necessary to say that $\alpha$ will be 10 based on the fundamental test.

\subsubsection{Stylistic Effects of Translation}

Stylistic effects in translation refer to the voice, order of the words, metaphors, and the pitch of the text. Whenever a translator wants to render a poem, he or she should observe the tone, metaphors, and the voice of the source and target language. In all above-mentioned cases, word order of each language is of great importance. As earlier explained, the system of word order in English would be SVO and the Japanese language would be SOV. How can Machine Translation distinguish the correct word order of the intended language? For example, the English sentence as "Advances in technology created new opportunities" was translated Japanese by Machine Translation as "Technology has advanced. There are new opportunities (John Hutchins, 2007, p5)." Japanese Machine Translation turned the English sentence into two parts. Each part has own subject and the verb. Or as another example, cliché, temporary, and stock metaphors are other problem faced by the Machine Translation. In most of the case, MT renders literally without paying attention to the content and degree of intensification of the intended metaphor. For example, " $a$ drain on resources" (Newmark, 1988, p. 109) can be rendered as "Una Perdita di Risorse" in the Italian language or "Unsere Mittel belasten" in the German language by Machine Translation which are completely literal and word for word translation (one to one correspondence). Therefore, in all these cases, post editing stage of Machine Translation is of great importance to convey the near essence of the source language. Last but not least, human assistance is an indispensible part of Machine Translation to transfer and interpret the source language text accurately.

\section{Suggestion}

To put it in the nutshell, one can say that none of the current Machine Translation models can solve all problems encountered during rendering. Therefore, one model should be used which combines all the above-mentioned models in it and utilizes them correctly to fill the lacunae in Machine Translation model. John Hutchins (2007) proposed "Hybrid Architectures" in that it covers (1) Rule-based Machine Translation for the source language analysis, (2) Example-based Machine Translation for transferring the source language to the target one, and (3) Statistics Machine Translation for target language generation in Interlingua stage of MT. It can be stated that Hybrid Machine Translation systems are parallel systems with the selection mechanism. The figure of Hybrid Machine Translation is as follows:

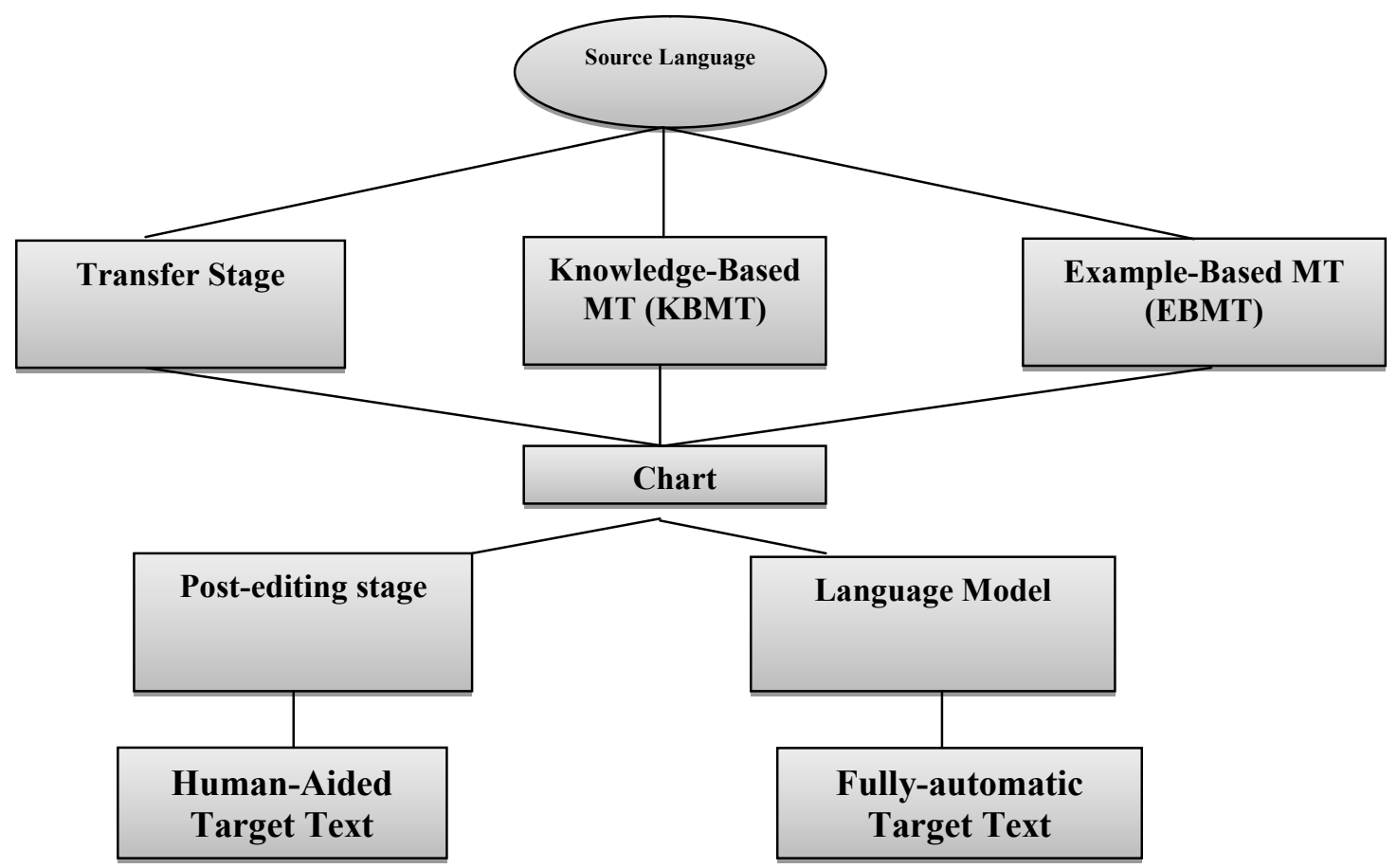

Figure2. Hybrid Architecture 


\section{Discussion}

Machine Translation nowadays is the important issue to be discussed. As a matter of fact, one can express that the future of the translation will turn to be automated. But, it should be noted that Machine Translation is part of picture and needs human assistance to work properly. This paper showed that the best method for rendering a text by Machine translation would to utilize the "Hybrid Architectures" and makes in one continuous process. In this connection, Translation Memory tools will have strong position in Machine Translation process. Totally, one can expect some future development from Machine Translation: (1) Integration of Translation Memory and Machine Translation. This kind of amalgamation causes the creation of huge lexicon databases for Machine Translation and it makes Machine Translation render correctly due to the diverse contextual factors. It can be stated that SYSTRAN is equipped with huge lexicons but low level of syntactic structures. (2) Amelioration of other Machine Translation Systems. It is better to say that Machine Translation metrics are heavily relied upon the traditional method of statistics and it is the time to delve into new metric and new method of mechanized translation. For example, Vít Baisa (2009) proposed a new method of Machine Translation Evaluation called "LAMENT" (LAnguage model and Meaning based Evaluation of machiNe Translation). (3) Quality improvement in translation. As earlier explained, Machine Translation is not able to determine and evaluate stylistic factors especially kinds of metaphors, idioms, and colloquial expressions in different situations. Most of the time, Machine Translation is going to deal with natural equivalence and one to one correspondence translation. (4) Norm-referenced Translation Evaluation. In this process, it is better to make one especial metric to evaluate the best translations amongst the worst ones. Norm-referenced translation evaluation provides this opportunity to render correctly and to transfer the content of the original text in the target language. (5) Scrutiny of Minority languages. Unfortunately, Machine Translation is used for major commercial languages and the language of military interest. It will be better to devise a Machine Translation which equips with minor languages and minor cultures due to the fact that every culture and language has its own background and structural systems. (6) Functionality which is of great importance in translation. Machine Translation should consider function, purpose, and domain of the source and target of the text. According to Skopos theory, the translator should satisfy the needs and preferences of the clients. Machine Translation should render in such a way that it can keep two poles in translation product altogether (Translator and Client). (7) Multilingual system of translation. It is prognosticated that in near future Machine Translation method operates on multilingual system of translation and then it can transfer of critical discourse analysis (CDA) wholly in different contexts of study. For example, in political speech or legal speech, Machine Translation should be able to act properly and consider all aspects of translation.

Last but not least, it is important to say that this study has been based on traditional metrics of Machine Translation and does not show any corresponding and new metric in this connection. It is hoped that some studies would address the case with other languages (Multilingual communication) and would accordingly set out to test the workability of the Machine Translation methods on a larger number of lexicon in the near future.

\section{Conclusion}

Translation covers various fields of study including linguistic, philosophy, sociology, and localization knowledge. This field involves semantics, syntax, and morphology. In all these cases, the translator should adopt an appropriate method of translation in order to convey the main essence of the source text to that of the target language. But in the route of translation, the translator may encounter numerous problems in transferring the content. It should be noted that one of the most influential instruments which can help human to translate will be Machine Translation. Machine Translation deals with linguistic knowledge of the source language and it cannot deal with translation strategies to meet the needs of the reader. In this direction, Machine Translation is not capable of interacting with large number of ambiguous tasks and connotational meaning. Therefore, human assistance is of great important in Machine Translation field. Generally, Human and Machine Translation's interaction are mutually exclusive. Last but not least, this paper scrutinized overall perspective of Machine Translation models and its metrics. Nowadays, Machine Translation is the key focus in Translation Studies and Evaluation for which Machine Translation is so useful when it deals with speed and money. In this paper, all of Machine Translation models and their metrics explained and the author was trying to transfer the whole idea completely. As a matter of fact, man-made instruments are not free of errors and it will be better to peruse the lacks and shortcomings correctly. Therefore, this study inspected the shortcomings of Machine Translation models in detail. And finally, it can be stated that current technologies are not progressively developed and need human interaction to do well in every situations.

\section{References}

Akbari, A. R. (2011). Intermediacy Model of Translation in Gray Zone, Oxford University Press, Unpublished Book.

Akbari, A. R. (2013). Exploring into the New Model Procedure in Translation: Wafting as a Case in Point. International Journal of Education and Literary Studies, 1, 19-20. Doi: 10.7575/aiac.ijels.v.1n.2p.18, http://dx.doi.org/10.7575/aiac.ijels.v.1n.2p.18

Baisa, V. (2009). Problems of Machine Translation Evaluation. Proceedings of Recent Advances in Slovanic Natural Language Processing, 21.

Bar-Hillel, Y. (1953). Some Linguistic Problems Connected with Machine Translation. Proceedings of Philosophy and Science, 20, 217-24. 
Cheragui. (2012). Theoretical Overview of Machine Translation. Proceeding ICWIT, 165-8.

Hatim, B. and I. Mason. (1997). The Translator as Communicator. London Routledge, 147.

Homedian, A. H. (1998). Machine Translation. Journal of King Saud University. Language and Translation, $10,1-21$.

Hutchins, J. (2007). Machine Translation: Problems and Issues, 1-7.

Kano, T. (2012). A Method for Translation of Paralinguistic Information. Proceeding of the $9^{\text {th }}$ International Workshop on Spoken Language Translation, 159-60.

IImo, A. (2003). Machine Translation, Research Seminar on Software Business, 15.

Munday, J. (2004). Introducing Translation Studies. London Routledge, 11.

Newmark, Peter. (1988). Textbook of Translation. U. K: Prentice Hall, 109.

Nida, E. A. (1964). Towards a Science of Translating. Leiden: E. J. Brill, 156-171.

Nirenburg, S. (1992). Multi-Purpose Development and Operation Environment for Natural Language Generation. Proceedings of the $3^{\text {rd }}$ Conference on Natural Language Application, Trento, Italy, 48.

Papineni, K. (2002). BLEU: A Method for Automatic Evaluation of Machine Translation. Proceedings of the 40 ${ }^{\text {th }}$ Annual Meeting of Association for Computational Linguistics (ACL), Philadelphia, 311-18.

Popovic, M and Ney, H. (2007). Word Error Rate: Decomposition over POS Classes and Application for Error Analysis. Proceedings of ACL Workshop on Machine Translation, 49-51.

Snover, M. (2006). A Study of Translation Edit Rate with Targeted Human Annotation. Proceeding of AMTA, Boston, 223-231.

Somers, H. (1998). "Machine Translation : History”, in M. Maker (ed.).

Tillman, C. (1997). Accelerated DP-Based Search for Statistical Translation. Proceedings of the $5^{\text {th }}$ European Conference on Speech Communication and Technology, 2667-70.

Webopedia. (2013). What is Natural Language? A Word Definition from the Webopedia Computer Dictionary.

Webster, M. (2009). Merriam Webster Collegiate Dictionary .

Yule, G. (2006). The Study of Language. Cambridge Publication, 100.

\section{Notes}

Note1. Medium Restricted Translation (MRT) subdivided according to translation by machine and human, with further subdivisions according to whether the machine/ computer is working alone or as an aid to the human translator, to whether the human translation is written or spoken and to whether spoken translation (interpreting) is consecutive or simultaneous.

Note2. Decoding ability in any language involves at least four principal levels: (1) the capacity of children whose vocabulary and cultural experience are limited. (2) The double standard of capacity of new literates, who can decode oral message with facility but whose ability to decode written message is limited. (3) the capacity of average literate adult, who can handle both oral and written messages with relative ease; and (4) the unusually high capacity of specialists (doctors, theologians, philosophers, scientists, etc.), when they are decoding messages within their own areas of specialization

Note3. Mediation is defined as "the extent to which translator intervene in the transfer process, feeding their own knowledge and belief into processing the text."

Note4. The number of candidate translation words and then divided by the total number of words in candidate translation.

Note5. Different part of speech 